\title{
Integrated Open Loop Resonator Filter Designed with Notch Patch Antenna for Microwave Applications
}

\author{
D. Azra Awang Mat ${ }^{\star}$, N. Syuhada Hasim, Nurmiza Othman, Amira Amran, \\ D. Norkhairunnisa Abang Zaidel, A. S. Wani Marzuki, Shafrida Sahrani, \\ Kismet anak Hong Ping, Rohana Sapawi \\ Applied Electromagnetic Research Group Engineering, Faculty of Engineering, 94300 Kota Samarahan, \\ Sarawak, Malaysia, Telp: 082-583309/ Fax: 082-583410 \\ ${ }^{*}$ Corresponding author, e-mail: amdazra@unimas.my
}

\begin{abstract}
This paper presented the design of integrated open loop resonator bandpass filter with notch type antenna for the use in microwave applications. Chebyshev type filter is selected as the filter characteristics and cascaded design with the antenna to produce a single module, Integrated Filter Antenna (IFA). Special feature of the antenna is the implementation of notch on the patch antenna to improve the efficiency. IFA is then simulated in electromagnetic simulation tool, Agilent Advance Design System (ADS) version 2016 and measured using R\&S Vector Network Analyzer. It shows that the proposed IFA produced good measured return loss $>-30 \mathrm{~dB}$ with both vertical and horizontal gain of 9.11dBi and 8.01dBi respectively.
\end{abstract}

Keywords: integrated antenna, notch patch, return loss, gain

Copyright $\odot 2017$ Universitas Ahmad Dahlan. All rights reserved.

\section{Introduction}

Antenna and filter are the two irreplaceable passive components in RF/microwave front end [1]. They are always treated as an individual research and mostly connected via transmission lines which might increase loss and circuit area. Integrating both antenna and filter into a single subsystem would solve the current problem involving, impedance mismatch, reducing both loss and the size thus enhancing overall performance of the system. Dealing with the impedance mismatches is one of the crucial concerns that all the designers should take seriously in designing integrated filter-antenna due to separate interconnection between them that will contribute to extra impedance transformation needed [2].

Recent research shows that different researchers introduced different design and method on integrating filter and antenna. For example, the co-design approach is being presented in to achieve miniaturization, low cost and also providing high selectivity of signal in their proposed design [2]. Not only that, the cascaded approach or known as traditional method also still being used in integrating filter-antenna [3]. Thus, this paper briefly discuss the proposed design of integrated filter antenna (IFA) with the combination of open loop resonator bandpass filter and notch patch antenna, fabricated on 1.6um FR4 substrate for microwave applications.

\section{The Design of Integrated Filter Antenna}

\subsection{The Filter}

Open loop resonator bandpass filter (OLRF) is used as the filter for the proposed design with the method of filter synthesis [4]. In this project, the OLRF with Chebyshev's characteristic is proposed because this type of filter form a smaller transition region than the same order of the Butterworth filter which is at the expenses of the ripples in its pass band and very efficient to minimize the height of the maximum ripple thus allows some ripple in the pass band and stop band, thus, stepper cut-off frequency will be realized. OLR is one examples of the compact and miniature filter. It is composed of a microstrip line that having both end loaded with folded open stubs. Folded arms of open stub is generally not only for increasing the loading capacitance to 
the ground, but it is also used for producing cross coupling. In facts, this kind of resonator filter is definitely differ from the miniaturized hairpin resonator especially in terms of their concepts and purposes. The researchers stated that the cross-coupled structure will helps to achieve high selectivity characteristics with transmission zeros. Thus, it can improve the skirt rejection of the microstrip filter. Besides, filter with high selectivity would give the best performance with minimum insertion loss. Figure 1 shows the example of the OLRF designed with asymmetric feed line [5-6].

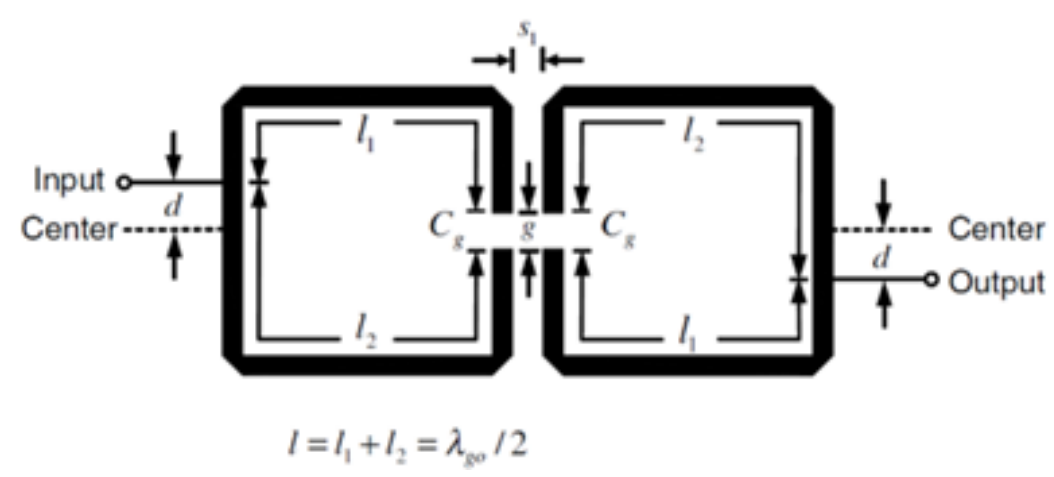

Figure 1. OLRF design with asymmetric feed line [5]

The equivalent circuit and lumped parameter circuit of OLR filter is shows in Figure 2. The Lumped parameter circuit of OLR filter shows the combination of low-pass (resonator A) and high-pass (resonator B) into a single filter which is bandpass filter.

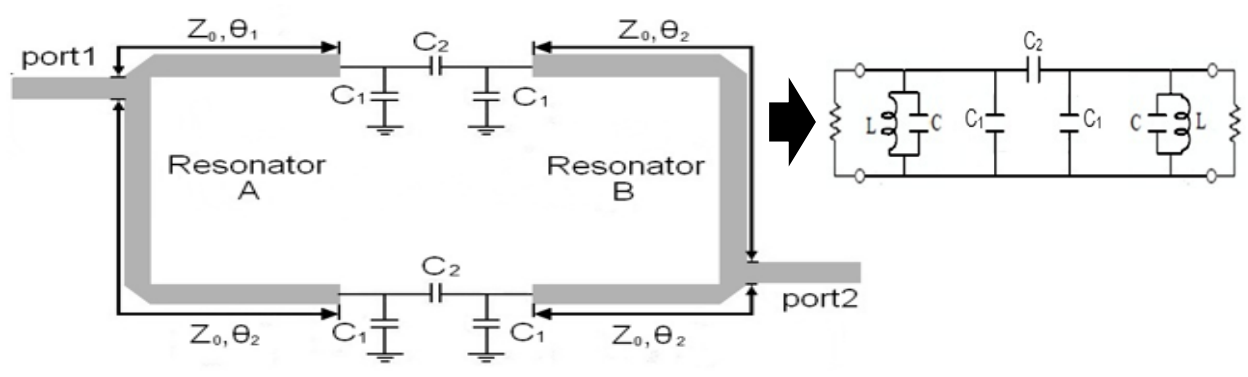

Figure 2. The equivalent circuit of Open-Loop Resonator filter and its lumped parameter circuit [5]

Recently, the researcher proved that the asymmetric structure gets the better out of the band rejection as compared to the symmetric structure for the two open-loop ring resonators and BPF will change its characteristics to high frequency if the width ratio of the filter $\left(W_{1} / W_{2}\right)$ is decrease. Besides, by reducing the gap, $(g)$ at the stub ended of the OLR, it will increase the coupling coefficient leading to narrow bandwidth. Besides, different position of the feed line also changed the filter's performance. Conventional filter suffered from high conductor loss and dielectric loss. To solve this, chamfered bend's structure is proposed in the filter as shown in Figure 4 to overcome discontinuities at microstrip bend that can contribute to error in phase and amplitude, thus decreasing the loss and increasing the performance of the design [7]. It is shown in [7], the selected chamfered bend produces better return loss of $3.87 \mathrm{~dB}$ at $2.31 \mathrm{GHz}$ compare to conventional chamfering as shown in Figure 3. The radius of the proposed chamfer bend is measured to be three times larger than the width of the resonators and by assuming the effective radius to be $R_{\text {eff }}=R_{\text {inner }}+0.3 W[8]$. 
Figure 3 shows the simulated results of $S_{21}$ of the proposed OLRF with $3.971 \mathrm{~dB}$. It proved that the insertion loss is reduced from $-6.2149 \mathrm{~dB}$ to $-3.971 \mathrm{~dB}$ due to chamfering and mitering techniques which helps to remove the excess capacitance at the corner of the bend.
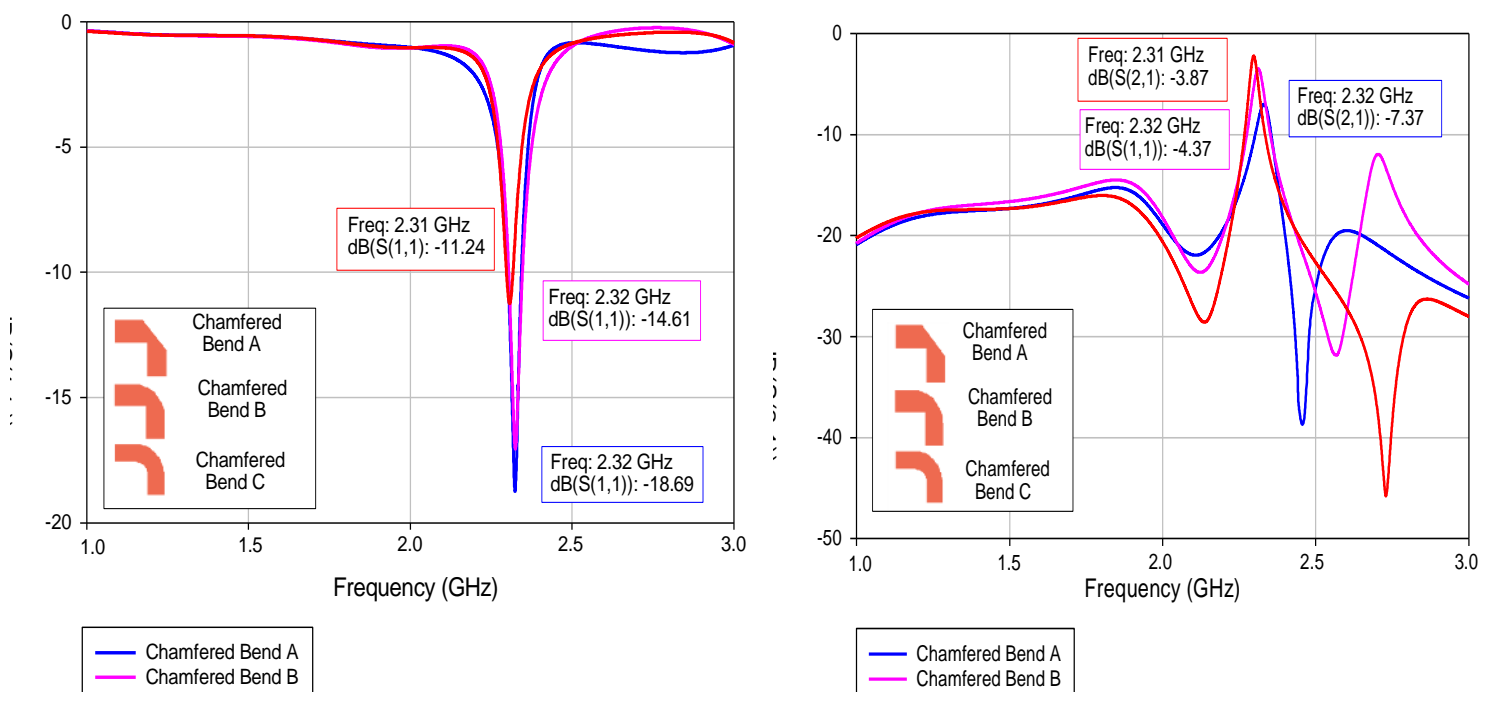

Figure 3. Return and Insertion Loss simulated results on different chamfering structure for the design of OLRBPF

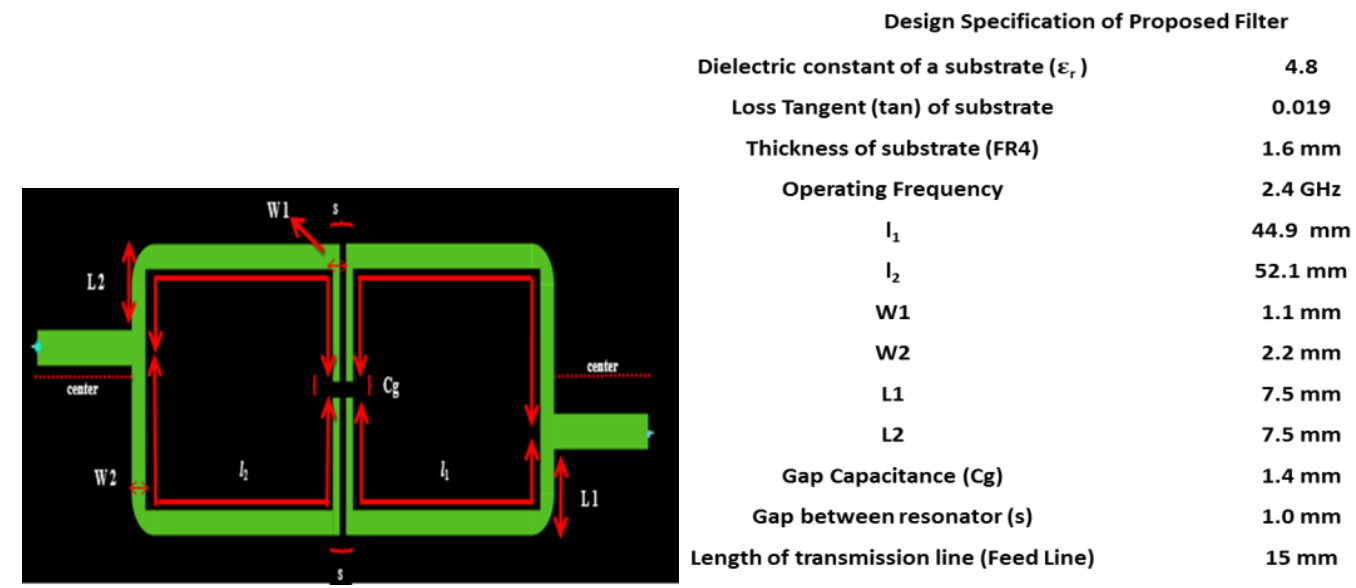

Figure 4. Proposed OLRF with chamfered bend and its specifications 


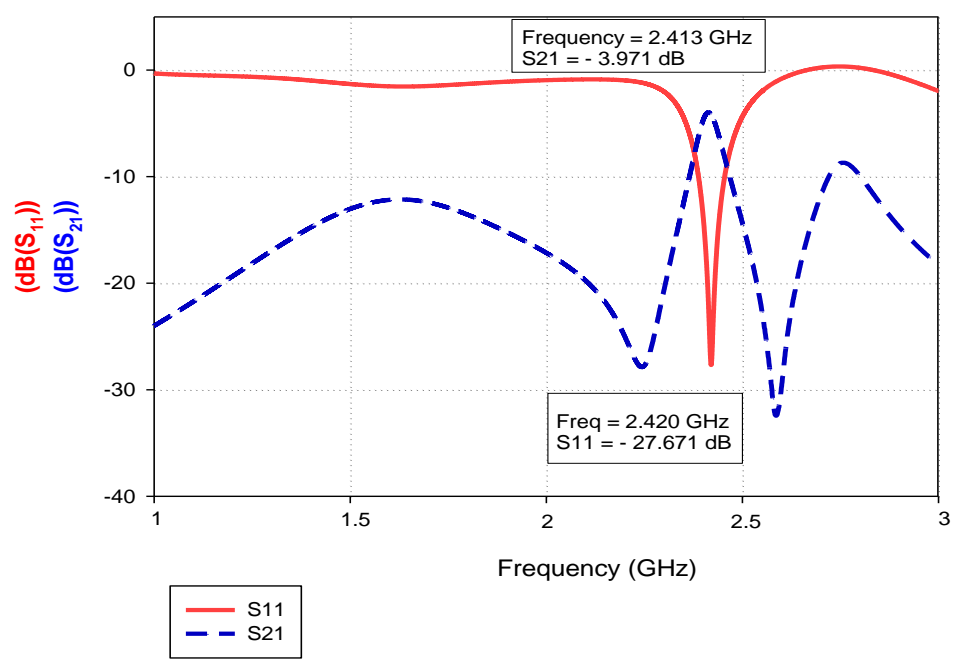

Figure 5. Simulated result of OLRF implemented with chamfered bends

\subsection{The antenna}

Normally, designing compact and small size microstrip patch antenna will give the drawbacks of narrow bandwidth [9-10]. Hence, notch-cut antenna and its detail specification has been proposed as shown in Figure 6.

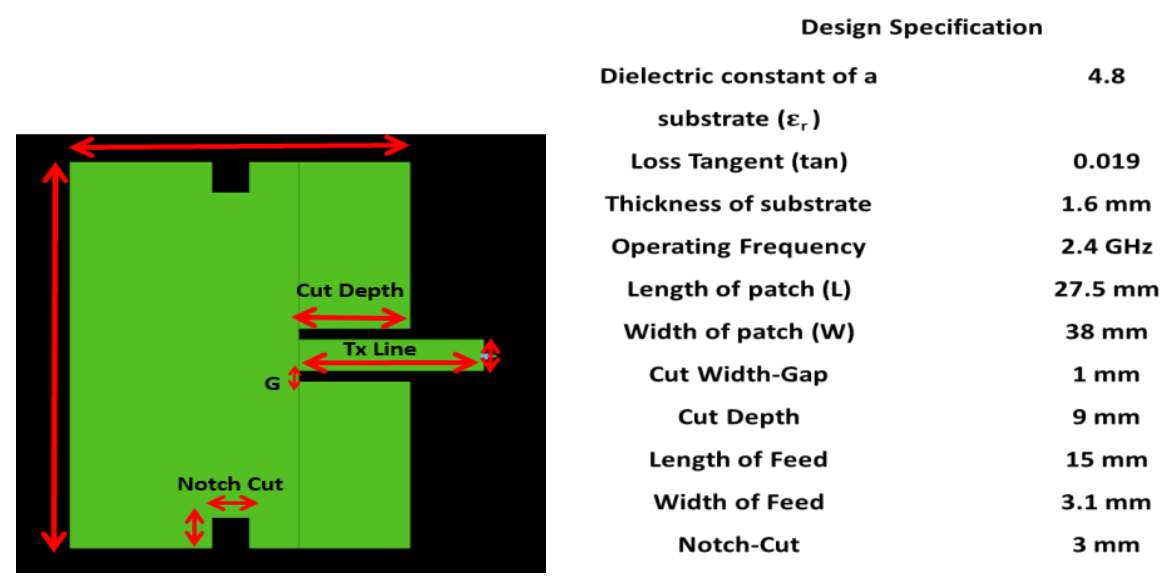

Figure 6. Proposed MPA with Pair of Notch Cut Specifications

Table 1 shows the comparison of bandwidth between conventional antennas with notch-cut MPA. It proved that the bandwidth of notch-cut antenna is improved to $1.77 \%$ as compared to the conventional MPA.

Table 1. Comparison of bandwidth between conventional MPA with notch-cut MPA

\begin{tabular}{lcc}
\hline & Conventional Antenna & Notch-Cut MPA \\
\hline FrequencyLow $\left(\mathrm{f}_{\mathrm{L}}\right)$ & $2.485 \mathrm{GHz}$ & $2.410 \mathrm{GHz}$ \\
Frequency High $\left(\mathrm{f}_{\mathrm{H}}\right)$ & $2.525 \mathrm{GHz}$ & $2.453 \mathrm{GHz}$ \\
Resonance Freguency $\left(\mathrm{F}_{\mathrm{c}}\right)$ & $2.505 \mathrm{GHz}$ & $2.429 \mathrm{GHz}$ \\
Bandwidth & $1.59 \%$ & $1.77 \%$ \\
\hline
\end{tabular}




\subsection{Integrated Filter Antenna (IFA)}

The OLRF are integrated with Notch-Cut MPA to achieve miniaturization and enhancing the bandwidth performances. The 3D layout is constructed and fabricated on FR4 dielectric substrates as shown in Figure 7.
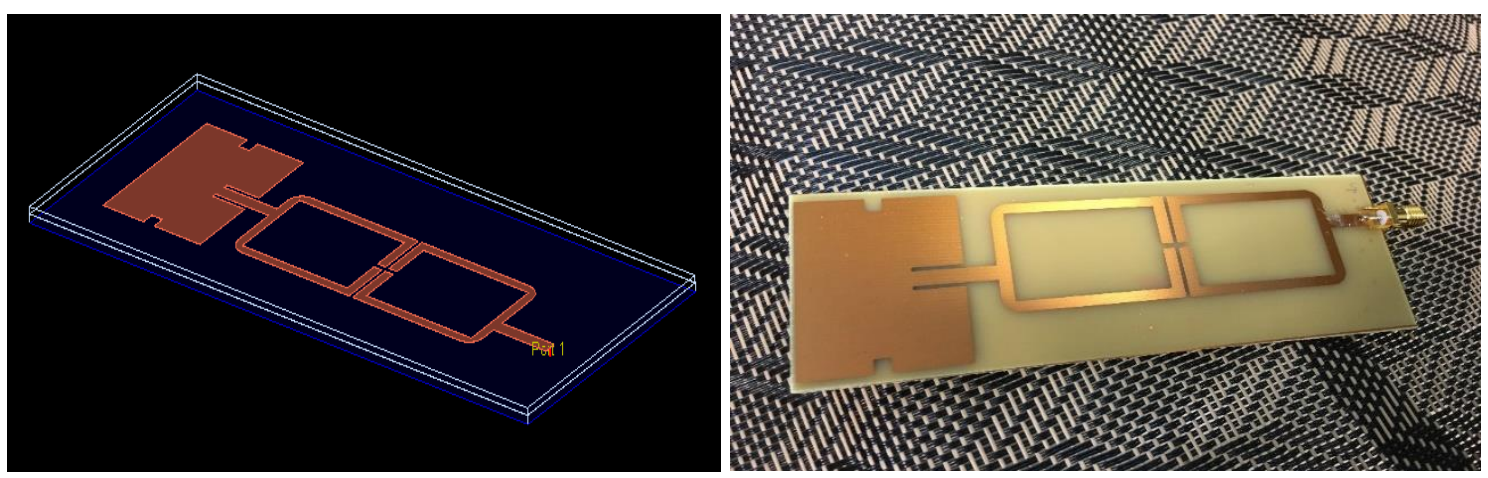

Figure 7. 3D Layout and fabricated design of OLR Filter integrated with notch-cut MPA in cascaded approach

Bandwidth for this type integration method system is about $8.78 \%$ as calculated. It is increases compared to the bandwidth of the single Notch-Cut MPA. As the bandwidth become wider, it allows to have many frequencies drops within the bandwidth range. Therefore, the limitation of using microstrip patch antenna that having narrow bandwidth are being solved after the filter structure are integrated.

\section{Measurements and Discussions}

Both measured and simulated results are collected for the proposed Integrated Filter Antenna (IFA) using R\&S Vector Network Analyzer and Electromagnetic Simulation Tools, Agilent Advanced Design System (ADS) 2006. From Figure 8, the simulated return loss, $\mathrm{S}_{11}$ falls at $-23.441 \mathrm{~dB}$ thus showing that the impedance matching are good for the proposed IFA. Since the resonance frequency drops from $2.40 \mathrm{GHz}$ to $2.30 \mathrm{GHz}$, it evidenced that the current path is longer due to the combination of notch-cut antenna and the OLRF[11]. Thus, the resonance frequency is reduced.

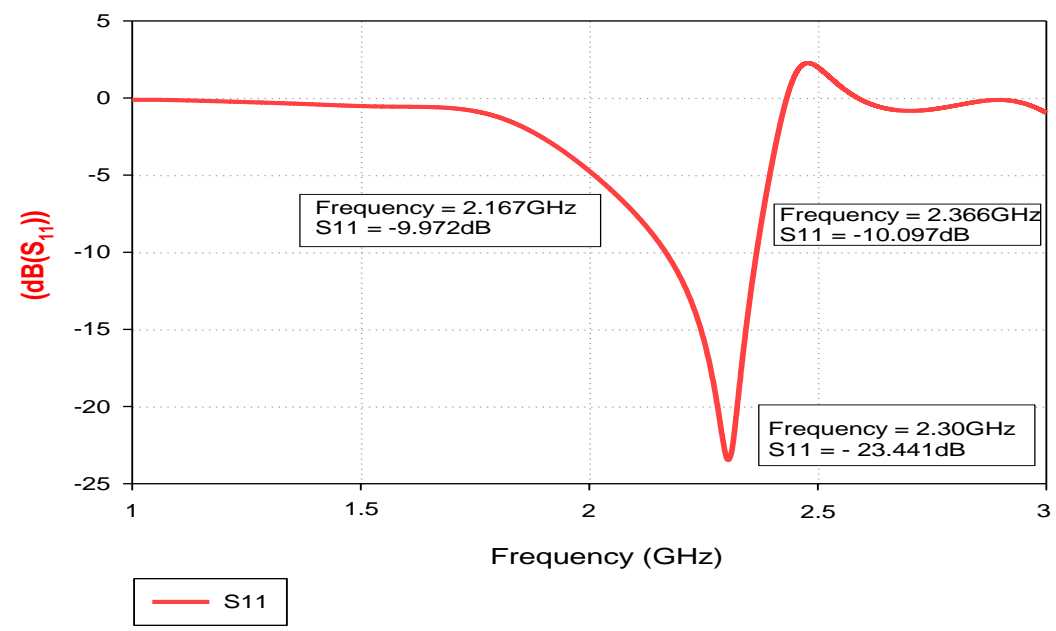

Figure 8. Simulated result of return loss, $S_{11}$ for proposed IFA 
Figure 9 shows the measured results for both return loss, $S_{11}$ and radiation pattern for the proposed IFA. It indicate both measured and simulated results falls at the same frequency of $2.36 \mathrm{GHz}$ with good impedance matching. Figure 9 shows both measured radiation pattern and gain for vertical gain of $9.11 \mathrm{dBi}$ and horizontal gain of $8.01 \mathrm{dBi}$ respectively.

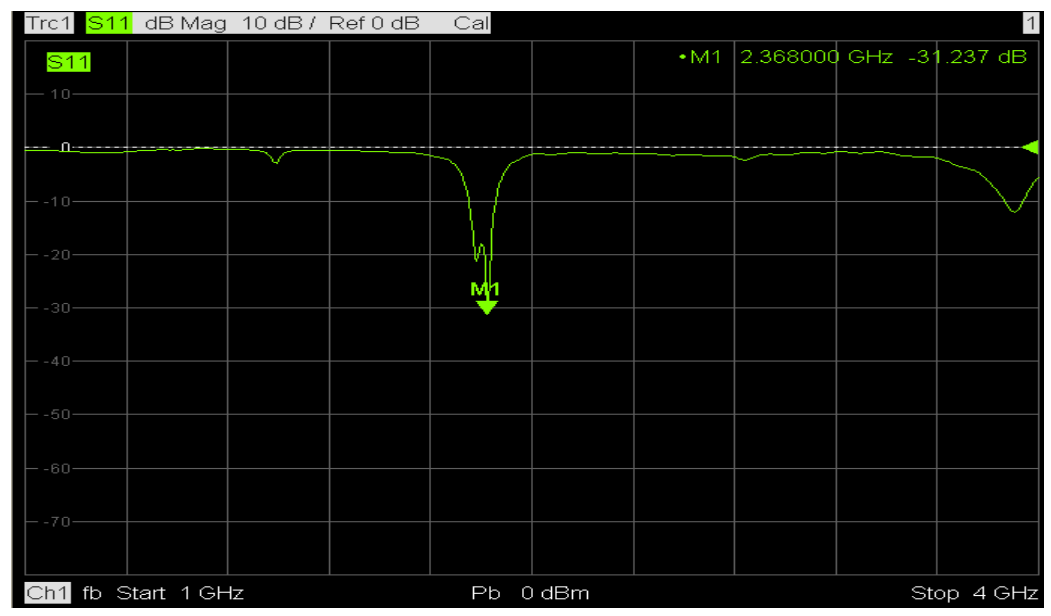

Figure 9. Measured $\mathrm{S}_{11}$
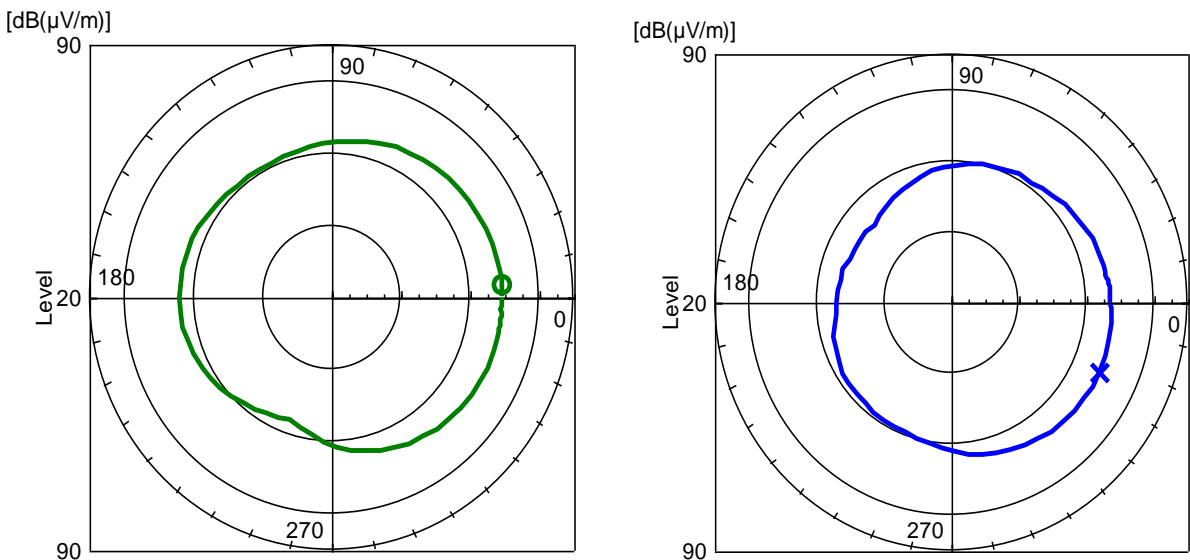

Vertical gain $=-9.11 \mathrm{dBi}$

Horizontal gain $=-8.01 \mathrm{dBi}$

Figure 10. Measured Horizontal and vertical antenna radiation pattern

\section{Conclusion}

One of the biggest and challenging task for the designer is to have an ideal integrated filter antenna to achieve miniaturisation and compact subsystem that having low insertion loss, high gain and wider bandwidth. Thus, in this research project, the introduction of OLRF with chamfered bend, Notch-cut MPA as well as integration method of cascaded and co-design will helps to achieve the target. The goal of this project is to integrate the filter and antenna into a single subsystem at the frequency of $2.4 \mathrm{GHz}$. It also to improve the performance of the integrated filter antenna in terms of their bandwidth, $S_{11}$ return loss parameter, gain and directivity as well as their radiation efficiency are achieved.

It is a crucial issue for the filter design to have high insertion loss. Therefore, OLRF with chamfered bend is designed in this project using parametric studies and several parameters that have been optimized to achieve a filter that can operate at $2.4 \mathrm{GHz}$. The introduction to the chamfered bend will act to reduce the effect of the discontinuity and proficient to increase the 
performance of the filter itself before being integrated with the antenna. OLRF with chamfered bend give the excellent performance as the $S_{11}$ values slightly higher than the $S_{11}$ of the conventional filter. The low loss of the OLRF with chamfered bend specifies that the overall loss is small because it is near to $0 \mathrm{~dB}$ as being compared to conventional filter.

In this research, microstrip patch antenna is used due to the fact that it has low cost, light weight and their nature of easy fabrication process. However, microstrip patch antenna suffers from narrow bandwidth. This problem cause a limitation on the performance of the microstrip. Therefore, notch-cut MPA is introduced. Notch-cut from the meandering techniques be able to enhance the bandwidth and reduced the resonance frequency in order to operate at $2.4 \mathrm{GHz}$. The bandwidth for the notch-cut antenna are increase compared to the conventional antenna. As for the gain, directivity and radiation efficiency, it shows that notch-cut antenna give the excellent performance.

With the emergences of the new technologies in communication, great demand of small size and light weight is increases. The integration between the filter and antenna are presented to have high performance of low insertion loss, low cost as well as to achieve the miniaturization. In this research, the analysis of cascaded and co-design integration method are being performed. Table 2 shows the summary of the performances for OLRF with chamfered bend, Notch-cut MPA as well as both integration method of cascaded and co-design in terms of $\mathrm{S}_{11}, \mathrm{~S}_{21}$ as well as its bandwidth.

Table 2. Comparison for OLRF with chamfered bend, Notch-cut MPA as well as both integration method of cascaded and co-design in terms of $S_{11}, S_{21}$ as well as its bandwidth

\begin{tabular}{llllll}
\hline & $\mathrm{S}(1,1)$ & $\mathrm{S}(2,1)$ & Bandwidth & $\begin{array}{l}\text { Resonance } \\
\text { Frequency }\end{array}$ & Size $(\mathrm{mm})$ \\
\hline OLRF with Chamfered Bend & $-27.671 \mathrm{~dB}$ & $-3.971 \mathrm{~dB}$ & & $2.420 \mathrm{GHz}$ & $95 \times 26$ \\
$\begin{array}{l}\text { Single Antenna - Notch-Cut } \\
\text { Microstrip Patch Antenna }\end{array}$ & $-24.723 \mathrm{~dB}$ & & $1.77 \%$ & $2.453 \mathrm{GHz}$ & $27.5 \times 38$ \\
$\begin{array}{l}\text { The proposed design - Integrated } \\
\text { Filter Antenna (Cascaded }\end{array}$ & $-23.441 \mathrm{~dB}$ & & $8.78 \%$ & $2.30 \mathrm{GHz}$ & $1140 \times 38$ \\
Approach) & & & & & \\
\hline
\end{tabular}

\section{Acknowledgements}

This work was supported Small Grant Scheme (SGS) from Universti Malaysia Sarawak with grant number SGS (F02(S181)/1325/2016)5)).

\section{References}

[1] Jadhav JB, Deore PJ. Filtering antenna with radiation and filtering functions for wireless applications. Journal of Electrical Systems and Information Technology, $9^{\text {th }}$ November 2016.

[2] Wei-Jun W, Qi-Feng L, Qi Zhang, Jing.-Ya D. Co-Design of A Compact Dual Band FilterAntenna for WLAN Application. Progress in Electromagnetics Research Letters. 2013; 40: 129-138.

[3] Zainuddin NIA. Integrated Filter Antenna for $2.4 \mathrm{GHz}$ Application. Final Year Thesis, Universiti Malaysia Sarawak; 2015.

[4] Mansour GM, Lancaster J, Hall PS, Gardner P, Nugoolcharoenlap E. Design of Filtering Microstrip Antenna Using Filter Synthesis Approach. Progress in Electromagnetics Research Letters. 2014; 145: 59-67.

[5] Awang Mat DA. Development of Slow Wave Structures for Millimeter-wave CMOS Applications. PhD Thesis, Kyushu University, Fukuoka, Japan; 2014.

[6] Hong J-S, Lancaster M.J. 2001. Microstrip filters for RF/Microwave Applications. John Wiley \& Sons.

[7] Noor NNM, Mat DAA, Amira A, Zaidel DKA, Jospeh A. The Effect of Chamfering Structure towards the Design of Open Loop Resonator Bandpass Filter for Microwave Applications. Journal of Telecommunication, Electronic and Computer Engineering. 2016; 8(12).

[8] Rosu I. Microstrio, Stripline, and CPW [Online]. Retrieved from http://www.qsl.net/va3iul/M icrostrip_Stripline_CPW_Design/Microstrip_Stripline_and_CPW_Design.pdf. 
[9] Kushwaha MS, Prasad RK. Improvement of Bandwidth of Microstrip Patch Antenna by Multiple Notches. Conference on Advances in Communication and Control System. 2013; 245-248.

[10] Balanis CA. Antenna Theory: Analysis and Design, Third Edit. A John Wiley \& Sons, Inc., Publication. 2005.

[11] Singh I, Tripathi V. Micro strip Patch Antenna and its Applications: A Survey. Int. J. Comp. Tech. Appl. 2011; 2(5): 1595-1599. 